\title{
Fractional Weak Discrepancy of Posets and Certain Forbidden Configurations
}

\author{
Alan Shuchat \\ Department of Mathematics \\ Wellesley College \\ Wellesley, MA 02481 USA \\ Randy Shull \\ Department of Computer Science \\ Wellesley College \\ Wellesley, MA 02481 USA \\ Ann N. Trenk* \\ Department of Mathematics \\ Wellesley College \\ Wellesley, MA 02481 USA
}

September 18, 2007

\begin{abstract}
In this paper we describe the range of values that can be taken by the fractional weak discrepancy of a poset subject to forbidden $\mathbf{r}+\mathbf{s}$ configurations, where $r+s=4$. Generalizing previous work on weak discrepancy in $[5,12,13]$, the notion of fractional weak discrepancy $w d_{F}(P)$ of a poset $P=(V, \prec)$ was introduced in [7] as the minimum nonnegative $k$ for which there exists a function $f: V \rightarrow \mathbf{R}$ satisfying (i) if $a \prec b$ then $f(a)+1 \leq f(b)$ and (ii) if $a \| b$ then $|f(a)-f(b)| \leq k$. Semiorders were characterized by their fractional weak discrepancy in [8]. Here we describe the range of values of $w d_{F}(P)$ according to whether $P$ contains an induced $\mathbf{2}+\mathbf{2}$ and/or an induced $\mathbf{3}+\mathbf{1}$. In particular, we prove that the range for an interval order that is not a semiorder (contains a $\mathbf{3}+\mathbf{1}$ but no $\mathbf{2}+\mathbf{2}$ ) is the set of rational numbers greater than or equal to one.
\end{abstract}

*Supported in part by a Wellesley College Brachman Hoffman Fellowship. 


\section{Introduction}

A weak order is a poset $P=(V, \prec)$ that can be assigned a real-valued function $f: V \rightarrow \mathbf{R}$ so that $a \prec b$ in $P$ if and only if $f(a)<f(b)$ [1]. Thus, the elements of a weak order can be ranked by a function that respects the ordering $\prec$ and issues a tie in ranking between incomparable elements $(a \| b)$. When $P$ is not a weak order, it is not possible to resolve ties as fairly. The weak discrepancy of a poset, introduced in [13] as the weakness of a poset, is a measure of how far a poset is from being a weak order [5], [12]. In [7], the problem of determining the weak discrepancy of a poset was formulated as an integer program whose linear relaxation yields a fractional version of weak discrepancy given in Definition 1 below.

Definition 1 The fractional weak discrepancy $w d_{F}(P)$ of a poset $P=(V, \prec)$ is the minimum nonnegative real number $k$ for which there exists a function $f: V \rightarrow \mathbf{R}$ satisfying

(i) if $a \prec b$ then $f(a)+1 \leq f(b) \quad$ ("up" constraints)

(ii) if $a \| b$ then $|f(a)-f(b)| \leq k$. ("side" constraints) Such a function $f$ is called an optimal fractional weak labeling of $P$ (or of $V$ ).

As an example, consider the salary assignment problem described in [8]. A manager wishes to assign a salary $f(a)$ to each employee $a$ in her division in a fair way. She can partially order the employees in her division based on their value to the company. The "up" constraints ensure that a more valuable employee receives a higher salary. The "side" constraints are fairness conditions that restrict the salary discrepancies between incomparable employees. For a weak order, sets of pairwise incomparable employees (antichains) are assigned the same salary level and the fractional weak discrepancy is zero ( $k=0$ satisfies the definition). In general, with the appropriate choice of unit the manager can assign $f(a)$ according to Definition 1 . The $k$ in this definition is a measure of the fairness of the assignment.

Denote the disjoint union of two chains with $r$ and $s$ elements, respectively, by $\mathbf{r}+\mathbf{s}$. A number of important classes of posets can be characterized in terms of forbidden $\mathbf{r}+\mathbf{s}$ configurations. For example, linear orders are posets with no induced $\mathbf{1}+\mathbf{1}$, and it is not hard to show that weak orders are posets with no induced $\mathbf{2}+\mathbf{1}$ [1]. Posets with no induced $\mathbf{2}+\mathbf{2}$ and no induced $\mathbf{3}+\mathbf{1}$ are known as semiorders. By a theorem of Scott and Suppes [6], this class is equivalent to the class of unit interval orders, that is, posets which can be represented as follows: each element $x$ of the ground set $V$ is assigned a unit length interval $I_{x}$ on the real number line so that $x \prec y$ if and only if the interval $I_{x}$ is completely to the left of $I_{y}$. In [8] we show how we can use fractional weak discrepancy to characterize the class of semiorders. In particular we establish the following two results.

Theorem 2 ([8]) If $P$ is a semiorder then $w d_{F}(P)=\frac{r}{r+1}$ for some integer $r \geq 0$. Furthermore, for each integer $r \geq 0$, there exists a semiorder $P$ with $w \bar{d}_{F}(P)=\frac{r}{r+1}$. Equivalently, $\left\{w d_{F}(P): P\right.$ a semiorder $\}=\left\{0, \frac{1}{2}, \frac{2}{3}, \frac{3}{4}, \frac{4}{5}, \ldots\right\}$. 
Theorem 3 ([8]) If $P$ is a poset that is not a semiorder then $w_{F}(P)$ is a rational number that is at least one. Furthermore, for each rational number $q \geq 1$, there exists a poset $P$ (that is not a semiorder) with $w d_{F}(P)=q$. Equivalently, $\left\{w d_{F}(P): P\right.$ a poset that is not a semiorder $\}=\{q \geq 1: q \in \mathbf{Q}\}$.

Combining the results of Theorems 2 and 3, we obtain the following characterization of semiorders.

Corollary $4([8])$ A poset $P$ is a semiorder if and only if $w d_{F}(P)=\frac{r}{r+1}$ for some integer $r \geq 0$.

Posets possessing no induced $\mathbf{2}+\mathbf{2}$ and/or no $\mathbf{3}+\mathbf{1}$ have been studied extensively beyond the class of semiorders. Relaxing the requirement that the poset contain no $\mathbf{3}+\mathbf{1}$, but retaining our restriction on no induced $\mathbf{2}+\mathbf{2}$, yields the well-known class of interval orders. These are, by definition, posets in which each element $x$ can be assigned an interval $I_{x}$ on the real line so that $x \prec y$ if and only if $I_{x}$ lies completely to the left of $I_{y}$ [2]. Posets that are $\mathbf{3}+\mathbf{1}$ free but may or may not contain a $\mathbf{2}+\mathbf{2}$ are not as well known as either semiorders or interval orders, but have a number of important properties nevertheless. For example, Stanley's generalization of the chromatic polynomial is $s$-positive for the incomparability graph of such a poset $[4,11]$. Skandera characterized posets containing no induced $\mathbf{3}+\mathbf{1}$ in terms of their antiadjacency matrices and used this characterization to give a simple proof that the chain polynomial of such posets has only real zeros [10].

In this paper we study the fractional weak discrepancy of posets obtained by selectively relaxing the restrictions on induced $\mathbf{2}+\mathbf{2}$ and induced $\mathbf{3}+\mathbf{1}$. Together with Theorem 3, Theorem 10 will imply that the range of the fractional weak discrepancy function for interval orders (no induced $\mathbf{2}+\mathbf{2}$ ) that are not semiorders (contain an induced $\mathbf{3}+\mathbf{1}$ ) is precisely the set of all rational numbers greater than or equal to 1 . Indeed, Corollary 15 states that any poset with fractional weak discrepancy greater than 1 must contain a $\mathbf{3}+\mathbf{1}$. We also show that the range of $w d_{F}$ when an induced $\mathbf{2}+\mathbf{2}$ is present also depends on the presence of a $\mathbf{3}+\mathbf{1}$ : when $P$ contains no induced $\mathbf{3}+\mathbf{1}$ then $w d_{F}(P)=1$ and when it does contain a $\mathbf{3}+\mathbf{1}$ then the range is again the set of rationals that are at least 1 . These results are summarized in Table 1.

\section{Forcing cycles}

We begin with some definitions and preliminary results.

Definition 5 A forcing cycle $C$ of poset $P=(V, \prec)$ is a sequence $C: x_{0}, x_{1}, \ldots, x_{m}=$ $x_{0}$ of $m \geq 2$ elements of $V$ for which $x_{i} \prec x_{i+1}$ or $x_{i} \| x_{i+1}$ for each $i: 0 \leq i<m$. If $C$ is a forcing cycle, we write $\operatorname{up}(C)=\left|\left\{i: x_{i} \prec x_{i+1}\right\}\right|$ and $\operatorname{side}(C)=\mid\{i$ : $\left.x_{i} \| x_{i+1}\right\} \mid$.

In [2], forcing cycles are called picycles (preference-indifference cycles). 


\begin{tabular}{|l||c|c|}
\hline & No $\mathbf{3}+\mathbf{1}$ & Yes $\mathbf{3}+\mathbf{1}$ \\
\hline & semiorders \\
$\begin{array}{l}\text { No } \mathbf{2}+\mathbf{2} \\
\text { interval orders }\end{array}$ & $\begin{array}{c}\left\{w d_{F}(P)\right\}=\left\{\frac{r}{r+1}\right\} \\
(\text { Corollary } 4)\end{array}$ & $\begin{array}{c}\left\{w d_{F}(P)\right\}=\{\text { rationals } \geq 1\} \\
\text { (Theorems 3, 10) }\end{array}$ \\
\hline Yes $\mathbf{2}+\mathbf{2}$ & $\begin{array}{c}w d_{F}(P)=1 \\
(\text { Corollary 16) }\end{array}$ & $\begin{array}{c}\left\{w d_{F}(P)\right\}=\{\text { rationals } \geq 1\} \\
\text { (Theorem 9; Corollary 15; Figure 3) }\end{array}$ \\
\hline
\end{tabular}

Table 1: Summary of results for the range of $w d_{F}$.

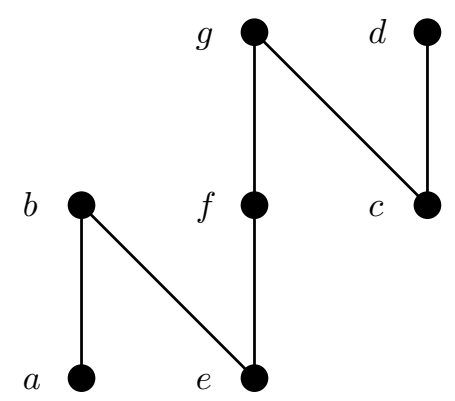

Figure 1: A poset with similar forcing cycles starting at $a$ with type $[2,2,3]$ and at $e$ with type $[3,2,2]$.

Let $C$ be a forcing cycle as in Definition 5. We may choose to start the cycle at an element $x_{0}$ that is the beginning of a sequence of "up" steps, i.e., $x_{0} \prec x_{1}$ and $x_{m-1} \| x_{m}=x_{0}$. We call $x_{0}$ an upward starting point of $C$. In this case, $C$ consists of $s$ successive chains of $a_{i} \geq 1$ elements each followed by an incomparability, $i=1,2, \ldots, s$, where $\sum_{i=1}^{s} a_{i}=m$. We write type $(C)=$ $\left[a_{1}, a_{2}, \ldots, a_{s}\right]$.

For example, the poset $P$ in Figure 1 has forcing cycle $C: a \prec b\|c \prec d\|$ $e \prec f \prec g \| a$ with $\operatorname{up}(C)=4$, side $(C)=3$ and type $(C)=[2,2,3]$. In general, a forcing cycle $C$ with type $(C)=\left[a_{1}, a_{2}, \ldots, a_{s}\right]$ has up $(C)=\sum_{j=1}^{s}\left(a_{j}-1\right)$ and side $(C)=s$. Note that $a_{i}=1$ corresponds to two consecutive incomparabilities in the forcing cycle.

Given a forcing cycle $C$, we can obtain a closely related forcing cycle $C^{\prime}$ by choosing a different upward starting point. For example, in Figure 1 we can start the forcing cycle at $e$ instead of $a$. Then we obtain $C^{\prime}: e \prec f \prec g \| a \prec$ $b\|c \prec d\| e$, which has type $\left(C^{\prime}\right)=[3,2,2]$.

Note that if $P$ has no incomparable pair then it is a linear order, has no forcing cycle, and $w d_{F}(P)=0$. The following result characterizes fractional 


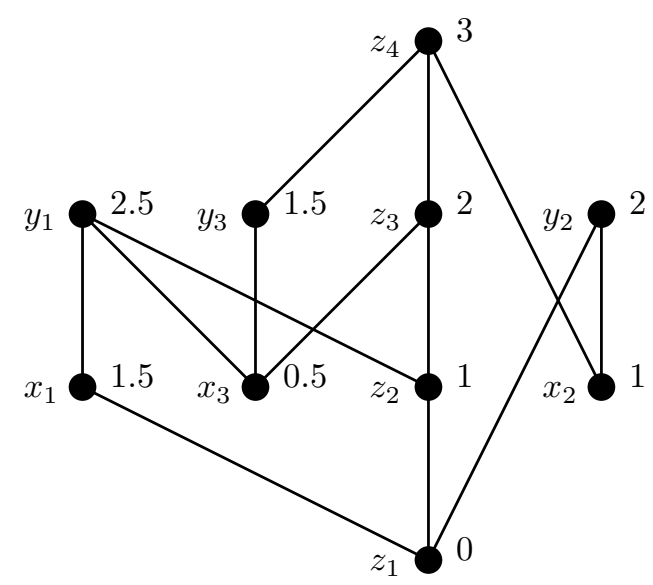

Figure 2: A poset with $w d_{F}(P)=\frac{3}{2}$ but no forcing cycle with $\operatorname{up}(C)=$ $3, \operatorname{side}(C)=2$.

weak discrepancy in terms of forcing cycles when $P$ has an incomparable pair. The analogous result for weak discrepancy appears in [5].

Theorem $6([7])$ Let $P=(V, \prec)$ be a poset with at least one incomparable pair. Then $w d_{F}(P)=\max _{C} \frac{\mathrm{up}(C)}{\operatorname{side}(C)}$, taken over all forcing cycles $C$ in $P$.

The proof of Theorem 3 shows that for integers $r \geq s \geq 2$, if $q=\frac{r}{s}$ (not necessarily in lowest terms) then there exist a non-semiorder $P$ with $w d_{F}(\stackrel{s}{P})=q$ and a forcing cycle $C$ in $P$ with $\operatorname{up}(C)=r, \operatorname{side}(C)=s$. It is thus natural to conjecture that for integers $r \geq s \geq 2$, every poset $P$ with $w d_{F}(P)=\frac{r}{s}$ has a forcing cycle $C$ with up $(C)=r$ and $\operatorname{side}(C)=s$. This is not the case even if $\frac{r}{s}$ is in lowest terms, as the following proposition shows.

Proposition 7 There exists a poset $P$ with $w d_{F}(P)=\frac{3}{2}$ but no forcing cycle $C$ with $\operatorname{up}(C)=3$ and $\operatorname{side}(C)=2$.

Proof. We show that the poset $P$ in Figure 2 has the desired property. By Definition 1, the labeling function shown there implies that $w d_{F}(P) \leq \frac{3}{2}$. By Theorem 6 the forcing cycle

$$
x_{1} \prec y_{1}\left\|x_{2} \prec y_{2}\right\| x_{3} \prec y_{3}\left\|z_{1} \prec z_{2} \prec z_{3} \prec z_{4}\right\| x_{1}
$$

shows that $w d_{F}(P) \geq \frac{6}{4}=\frac{3}{2}$, thus $w d_{F}(P)=\frac{3}{2}$.

It is easy to see that there is no $4+\mathbf{1}$ in $P$ because there is only one chain of four elements, $z_{1} \prec z_{2} \prec z_{3} \prec z_{4}$, and every other element in $P$ is comparable to 
some $z_{i}$. Similarly, one can check that there is no $\mathbf{3}+\mathbf{2}$ in $P$ by considering all possible chains of three elements. This implies that $P$ cannot contain a forcing cycle $C$ with up $(C)=3$ and $\operatorname{side}(C)=2$ because we could choose an upward starting point for such a cycle to yield one of type $[4,1]$, a $\mathbf{4}+\mathbf{1}$, or of type $[3,2]$, a $\mathbf{3}+\mathbf{2}$.

Lemma 8, which appears as Proposition 9 of [8], allows us to describe optimal fractional weak labelings for forcing cycles whose "up" to "side" ratios achieve the maximum value of $w d_{F}(P)$. In particular, every optimal labeling is tight on such a forcing cycle in the following sense.

Lemma 8 ([8]) Let $C: x_{0}, x_{1}, \ldots, x_{m-1}, x_{m}=x_{0}$ be a forcing cycle for poset $P=(V, \prec)$ such that $k=w d_{F}(P)=\frac{u p(C)}{\operatorname{side}(C)}$ and let $f: V \rightarrow \mathbf{R}$ be an optimal fractional weak labeling of $P$. For each $i \in\{0,1, \ldots, m-1\}$

(i) if $x_{i} \prec x_{i+1}$ then $f\left(x_{i}\right)+1=f\left(x_{i+1}\right)$

(ii) if $x_{i} \| x_{i+1}$ then $f\left(x_{i+1}\right)-f\left(x_{i}\right)=-k$.

For example, the labeling shown in Figure 2 is tight on the forcing cycle given in the proof of Proposition 7.

\section{The Range of $w d_{F}$ and Interval Orders}

In Theorem 3 we find the range of the fractional weak discrepancy function for posets that are not semiorders. In this section we divide the non-semiorders into two types and find the range for each: non-interval orders and interval orders that are not unit interval orders.

Theorem 9 If $P$ is a non-interval order, then $w d_{F}(P) \geq 1$. Furthermore, for any rational number $q \geq 1$, there exists a non-interval order $P$ with $w d_{F}(P)=q$. Thus for the class of non-interval orders, the range of $w d_{F}$ is $\{q \in \mathbf{Q}: q \geq 1\}$.

Proof. If $P$ is not an interval order (i.e., possesses an induced $\mathbf{2}+\mathbf{2}$ ) then $P$ is not a semiorder, so $w d_{F}(P) \geq 1$ by Theorem 3 .

Now let $q>1$ be rational. The proof of Theorem 3 includes the construction of a poset $P$ with $w d_{F}(P)=q$. This construction, which appears in Proposition 14 of [8], contains an induced $\mathbf{2}+\mathbf{2}^{1}$, so $P$ is not an interval order.

For the case of $q=1$ we consider Figure 3 , which gives a poset $P$ containing an induced $\mathbf{2}+\mathbf{2}$, so again $P$ is not an interval order. The labeling of $P$ shown in the figure demonstrates that $w d_{F}(P) \leq 1$. On the other hand $C: a \| c \prec$ $d \prec e \| a$ is a forcing cycle with $\operatorname{up}(C)=\operatorname{side}(C)=2$, so $w d_{F}(P)=1$.

We now establish a similar result for interval orders. In particular, we show how to achieve any rational number that is at least one as the fractional weak discrepancy of some interval order, which by Theorem 3 is necessarily not a semiorder. The proof is constructive.

\footnotetext{
${ }^{1}$ The $\mathbf{2}+\mathbf{2}$ is formed by the chains $x_{n-1} \prec y_{n-1}$ and $z_{1} \prec z_{2}$.
} 


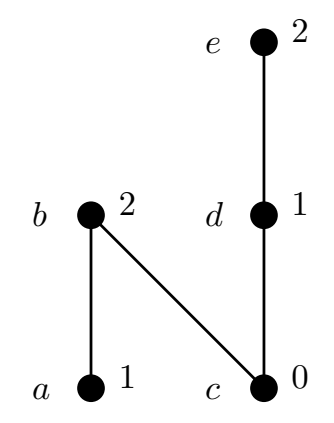

Figure 3: A non-interval order $P$ with $w d_{F}(P)=1$.

Theorem 10 For any rational number $q \geq 1$, there exists an interval order $P$ with $w_{F}(P)=q$.

Proof. We write the given rational $q$ as $q=r / s$ with integers $r \geq s \geq 2$. We will construct an interval representation of an order $P=(V, \prec)$ with $V=$ $\left\{x_{0}, x_{1}, \ldots, x_{r}, y_{1}, y_{2}, \ldots, y_{s-1}\right\}$. For $0 \leq i \leq r$, let $I\left(x_{i}\right)=[i s, i s]$, that is, each of these intervals is a point. Let $I\left(y_{s-1}\right)=[(s-2) r, s r]$ and if $s>2$, then for $1 \leq j \leq s-2$, let $I\left(y_{j}\right)=\left[(j-1) r,(j+1) r-\frac{1}{2}\right]$. Figure 4 shows the representation in the case $r=7$ and $s=4$. By construction, $x_{i} \prec x_{i+1}$ for $0 \leq i<r$ and $y_{j} \| y_{j+1}$ for $1 \leq j \leq s-2$. Furthermore, $x_{r} \| y_{s-1}$ and $y_{1} \| x_{0}$. Thus $P$ contains the forcing cycle $C: x_{0} \prec x_{1} \prec x_{2} \prec \cdots \prec x_{r}\left\|y_{s-1}\right\| y_{s-2}\|\cdots\| y_{1} \| x_{0}$ with $\operatorname{up}(C)=r$ and side $(C)=s$. Thus $w d_{F}(P) \geq r / s$ by Theorem 6 .

It remains to show $w d_{F}(P) \leq r / s$. Define the labeling function $g: V \rightarrow \mathbf{Z}$ by setting $g\left(x_{i}\right)=i s$ for $i=0,1, \ldots, r$ and setting $g\left(y_{j}\right)=j r$ for $j=1,2, \ldots, s-1$. (See the example in Figure 4.) We show

(i) if $a \prec b$ then $g(a)+s \leq g(b)$

(ii) if $a \| b$ then $|g(a)-g(b)| \leq r$.

("up" constraints) ("side" constraints) Then it will follow that the function $f: V \rightarrow \mathbf{Q}$ defined by $f(x)=g(x) / s$ is an optimal fractional weak labeling of $P$ satisfying Definition 1.

We will consider all pairs $(a, b)$ of elements of $V$, classify their relation in the poset, and prove that the corresponding constraints are satisfied. First take $x_{i}, x_{j} \in V$ with $i<j$. By construction, $x_{i} \prec x_{j}$ and $g\left(x_{i}\right)+s=i s+s=$ $(i+1) s \leq j s=g\left(x_{j}\right)$, satisfying (i) for this pair of elements.

Next consider $y_{i}, y_{j} \in V$ with $i<j$. If $j=i+1$, then $I\left(y_{i}\right) \cap I\left(y_{j}\right) \neq \emptyset$ so $y_{i} \| y_{j}$ and $\left|g\left(y_{i}\right)-g\left(y_{j}\right)\right|=|i r-j r|=r$, satisfying (ii). Otherwise, $j \geq i+2$. Let $R$ be the right endpoint of the interval $I\left(y_{i}\right)$ and $L$ be the left endpoint of the interval $I\left(y_{j}\right)$. Then $R=(i+1) r-\frac{1}{2} \leq(j-1) r-\frac{1}{2}<L$. Thus $y_{i} \prec y_{j}$ and $g\left(y_{i}\right)+s=i r+s \leq i r+r=(i+1) r<j r=g\left(y_{j}\right)$, satisfying (i). 


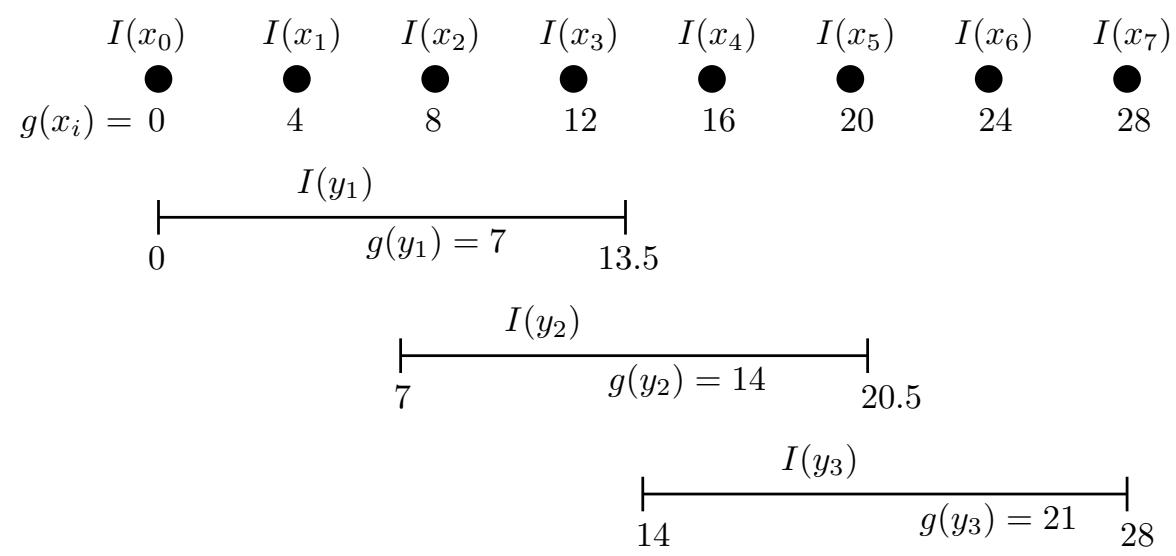

Figure 4: An interval order $P$ with $w d_{F}(P)=7 / 4$ and labeling function $g$.

Lastly, consider $x_{i}, y_{j} \in V$. By construction, $x_{i} \| y_{j}$ precisely when the point $I\left(x_{i}\right)$ is contained in the interval $I\left(y_{j}\right)$. In this case, for $1 \leq j \leq s-1$ we have $(j-1) r \leq i s \leq(j+1) r$. Subtracting $j r$ yields $-r \leq i s-j r \leq r$. Thus $\left|g\left(x_{i}\right)-g\left(y_{j}\right)\right|=|i s-j r| \leq r$, satisfying (ii).

If instead $x_{i} \prec y_{j}$ then the point $I\left(x_{i}\right)$ lies strictly to the left of $I\left(y_{j}\right)$, so $i s<(j-1) r$. In this case, $g\left(x_{i}\right)+s=i s+s<(j-1) r+s \leq(j-1) r+r=$ $j r=g\left(y_{j}\right)$, satisfying (i). Finally, if $y_{j} \prec x_{i}$ then $j<s-1$ and the point $I\left(x_{i}\right)$ lies strictly to the right of the interval $I\left(y_{j}\right)$, and thus $(j+1) r-\frac{1}{2}<i s$. Since all the parameters are integers, in fact, $(j+1) r \leq i s$. In this case $g\left(y_{j}\right)+s=$ $j r+s \leq j r+r=(j+1) r \leq i s=g\left(x_{i}\right)$, satisfying (i).

We can now fill in the top-right entry of Table 1 . If $P$ contains an induced $\mathbf{3}+\mathbf{1}$ but no $\mathbf{2}+\mathbf{2}$, then Theorem 3 implies $w d_{F}(P) \geq 1$. Conversely, by Theorem 10 any rational $q \geq 1$ equals $w d_{F}(P)$ for some interval order $P$ (contains no $\mathbf{2}+\mathbf{2}$ ), but Theorem 2 implies $P$ is not a semiorder (thus contains a $\mathbf{3}+\mathbf{1}$ ). We have shown the following.

Corollary 11 For the class of posets that are interval orders but not semiorders (contain an induced $\mathbf{3}+\mathbf{1}$ but no $\mathbf{2}+\mathbf{2}$ ), the range of $w d_{F}$ is $\{q \in \mathbf{Q}: q \geq 1\}$.

\section{An Upper Bound on $w d_{F}$ for Posets with no $\mathbf{n}+\mathbf{1}$}

As in $[7,8]$, we define the (integer) weak discrepancy $w d(P)$ of a poset $P=(V, \prec)$ as the minimum nonnegative integer $k$ for which there exists a function $f: V \rightarrow$ $\mathbf{Z}$ satisfying ( $i)$ and (ii) of Definition 1 . This is equivalent to the concept of weakness first introduced in [13]. The following theorem (Proposition 7 of [7]) 
allows us to calculate the weak discrepancy of a poset from its fractional weak discrepancy.

Theorem $12([7]) \quad$ For any poset $P$ we have $w d(P)=\left\lceil w d_{F}(P)\right\rceil$.

In [13], the author proved a result giving an upper bound on $w d(P)$ for posets with no induced $\mathbf{n}+\mathbf{1}$. We state the result in its contrapositive form.

Theorem $13([\mathbf{1 3}])$ Every poset $P$ with $w d(P)>n-2$ contains an induced $\mathbf{n}+\mathbf{1}$.

Neither forcing cycles nor fractional weak discrepancy had been defined when Theorem 13 was first presented. In this section, we give a substantially simpler proof of the analogous theorem for fractional weak discrepancy and show the two results are in fact equivalent.

Theorem 14 Every poset $P$ with $w d_{F}(P)>n-2$ contains an induced $\mathbf{n}+\mathbf{1}$.

Proof. Let $P=(V, \prec)$ be an interval order with $k=w d_{F}(P)>n-2$ and let $f: V \rightarrow \mathbf{R}$ be an optimal fractional weak labeling of $P$. By Theorem 6 there exists a forcing cycle $C: x_{0}, x_{1}, \ldots, x_{m}=x_{0}$ such that $k=w d_{F}(P)=$ $\frac{\operatorname{up}(C)}{\operatorname{side}(C)}$. Without loss of generality, suppose that $x_{m-1} \| x_{m}=x_{0}$, i.e., the cycle closes with an incomparability. By Lemma 8, the labeling $f$ is tight on $C$. In particular, $f\left(x_{m-1}\right)-f\left(x_{0}\right)=k$.

Consider the sequence of differences $S: f\left(x_{1}\right)-f\left(x_{0}\right), f\left(x_{2}\right)-f\left(x_{1}\right), \ldots, f\left(x_{m-1}\right)-$ $f\left(x_{m-2}\right)$. Note that the sum of the elements of $S$ is $f\left(x_{m-1}\right)-f\left(x_{0}\right)=k$. By Lemma 8, each term of $S$ is either +1 or $-k$. Let $t$ be the largest number of consecutive +1 's in $S$. If $t<k$ then every partial sum of $S$, and in particular the sum of all the terms, is less than $k$, a contradiction. Thus, $t \geq k$ and since $t$ is an integer, $t \geq\lceil k\rceil$. By the definition of $t$, there is a longest chain in $C$ containing $t$ elements. Let $x_{j}$ be its starting point and consider its subchain $x_{j} \prec x_{j+1} \prec \cdots \prec x_{j+\lceil k\rceil}$ of length $\lceil k\rceil$. By the maximality of $t, x_{j-1} \| x_{j}$ (if $j=0$ we replace $j$ by $m$ ) and thus $f\left(x_{j-1}\right)-k=f\left(x_{j}\right)$. Now

$$
f\left(x_{j+\lceil k\rceil}\right)=f\left(x_{j}\right)+\lceil k\rceil=f\left(x_{j-1}\right)-k+\lceil k\rceil<f\left(x_{j-1}\right)+1
$$

If $x_{j-1} \prec x_{j+\lceil k\rceil}$ then (1) contradicts the "up" constraint in Definition 1. If $x_{j+\lceil k\rceil} \prec x_{j-1}$ then $x_{j} \prec x_{j+\lceil k\rceil} \prec x_{j-1}$, contradicting $x_{j-1} \| x_{j}$. Thus, $x_{j-1} \| x_{j+\lceil k\rceil}$. We conclude that $x_{j} \prec x_{j+1} \prec \cdots \prec x_{j+\lceil k\rceil} \| x_{j-1}$ is a $(\lceil\mathbf{k}\rceil+\mathbf{1})+\mathbf{1}$. Since $k>n-2$ and $n-2$ is an integer, $\lceil k\rceil \geq n-1$ and $P$ contains an induced $\mathbf{n}+\mathbf{1}$.

The bound given in the Theorem 14 is the best possible, since $P=(\mathbf{n}-\mathbf{1})+(\mathbf{n}-\mathbf{1})$ has no induced $\mathbf{n}+\mathbf{1}$ but $w d_{F}(P)=\frac{2(n-2)}{2}=n-2$.

The hypotheses of Theorems 13 and 14 are equivalent because $n$ is an integer and $w d(P)=\left\lceil w d_{F}(P)\right\rceil$. Thus our proof of Theorem 14 gives a shorter proof of Theorem 13 as well. Notice also that the proof of Theorem 14 relies on the existence of a forcing cycle and an optimal labeling that is tight on that cycle. 
This same argument cannot be used to prove Theorem 13 directly since the tightness condition need not hold for forcing cycles whose "up" to "side" ratios achieve the (integer) weak discrepancy of the poset. For example, let $P$ be a $\mathbf{3}+\mathbf{2}$ with chains $a_{0} \prec a_{1} \prec a_{2}$ and $a_{3} \prec a_{4}$. An optimal integer labeling is $f\left(a_{0}\right)=0, f\left(a_{1}\right)=1, f\left(a_{2}\right)=2, f\left(a_{3}\right)=1, f\left(a_{4}\right)=2$ so $w d(P)=2$, but the labeling is not tight on the forcing cycle $a_{0} \prec a_{1} \prec a_{2}\left\|a_{3} \prec a_{4}\right\| a_{0}$.

\section{The Range of $w d_{F}$ and non-Interval Orders}

In Theorem 9 we find the range of the fractional weak discrepancy function for non-interval orders. In this section, we divide these orders into two types and find the range for each: orders that contain an induced $\mathbf{3}+\mathbf{1}$ and those that do not. This will justify the entries at the bottom of Table 1 .

The poset $P$ in Figure 2 has $w d_{F}(P)=3 / 2$ and contains no induced $\mathbf{4}+\mathbf{1}$ but it does have a $\mathbf{3}+\mathbf{1}$, e.g., the elements of $z_{1} \prec z_{2} \prec z_{3}$ are all incomparable to $x_{2}$. Indeed, all posets with fractional weak discrepancy greater than one must contain a $\mathbf{3}+\mathbf{1}$ by Theorem 14, with $n=3$. We state that specific case so we can refer to it more easily.

Corollary 15 Every poset $P$ with $w d_{F}(P)>1$ contains an induced $\mathbf{3}+\mathbf{1}$.

This result is best possible since, by Theorem 3, if $w d_{F}(P)<1$ then $P$ must be a semiorder and thus does not contain a $\mathbf{3}+\mathbf{1}$. On the other hand, [7] shows that $w d_{F}(\mathbf{3}+\mathbf{1})=w d_{F}(\mathbf{2}+\mathbf{2})=1$ so if $w d_{F}(P)=1$ then $P$ may or may not contain a $\mathbf{3}+\mathbf{1}$.

Note that Theorem 9 implies that the range of $w d_{F}$ for posets possessing an induced $\mathbf{2}+\mathbf{2}$ is the set of all rational numbers greater than or equal to 1 . In the case of strict inequality, Corollary 15 implies all such posets must also have an induced $\mathbf{3}+\mathbf{1}$. The poset $P$ given in Figure 3 possesses both a $\mathbf{2}+\mathbf{2}$ and a $\mathbf{3}+\mathbf{1}$ and has $w d_{F}(P)=1$. We conclude that the range of $w d_{F}$ for posets possessing both an induced $\mathbf{2}+\mathbf{2}$ and an induced $\mathbf{3}+\mathbf{1}$ is also $\{q \in \mathbf{Q}: q \geq 1\}$, as indicated in the lower-right entry in Table 1.

By Corollary 15 a poset $P$ with no induced $\mathbf{3}+\mathbf{1}$ must satisfy $w d_{F}(P) \leq 1$. Also, if $P$ has an induced $\mathbf{2}+\mathbf{2}$ then it has a forcing cycle $C$ with $\frac{\mathrm{up}(C)}{\operatorname{side}(C)}=1$ and thus by Theorem $6, w d_{F}(P) \geq 1$. So $w d_{F}(P)=1$, which fills in the lowerleft entry of Table 1 and which we state as an additional corollary. The converse is clearly false since $w d_{F}(\mathbf{3}+\mathbf{1})=1$. We have

Corollary 16 Every poset $P$ with an induced $\mathbf{2}+\mathbf{2}$ but no induced $\mathbf{3}+\mathbf{1}$ satisfies $w d_{F}(P)=1$.

Although Corollary 15 gives the best possible bound for $w d_{F}(P)$ over the class of all posets, the upper row of Table 1 suggests a slightly better bound when $P$ is restricted to the class of interval orders. In particular, if $C$ is a forcing cycle for $P$ with up $(C)>r$ and $\operatorname{side}(C)=r+1\left(\right.$ so $\left.w d_{F}(P)>\frac{r}{r+1}\right)$, then $C$ must contain a $3+\mathbf{1}$. Furthermore, the proof of Theorem 2 given in 
Proposition 16 of [8] shows how to construct, for each $r>0$, an interval order $P$ possessing an optimal forcing cycle $C$ with $\operatorname{up}(C)=r$ and $\operatorname{side}(C)=r+1$ but no induced $3+1$. In the case $n=3$, we can express the upper row as saying that if $\operatorname{up}(C)>(n-2) r$ and $\operatorname{side}(C)=r+1$, then $P$ must contain an $\mathbf{n}+\mathbf{1}$. In [9] we extend this result to the case where $n \geq 3$.

Acknowledgment. The third author feels very privileged to have collaborated with Peter Fishburn on papers [3] and [12].

\section{References}

[1] K.P. Bogart. Introductory Combinatorics. Harcourt Brace Jovanovich, New York, 1990.

[2] P.C. Fishburn. Interval orders and interval graphs: A study of partially ordered sets. John Wiley \& Sons, New York, 1985.

[3] P.C. Fishburn, P.J. Tanenbaum, and A.N. Trenk. Linear discrepancy and bandwidth. ORDER, 18:237-245, 2001.

[4] V. Gasharov. Incomparability graphs of $(\mathbf{3}+\mathbf{1})$-free posets are $s$-positive. Discrete Math., 157:211-215, 1996.

[5] J.G. Gimbel and A.N. Trenk. On the weakness of an ordered set. SIAM J. Discrete Math., 11:655-663, 1998.

[6] D. Scott and P. Suppes. Foundational aspects of theories of measurement. J. Symbolic Logic, 23:113-128, 1958.

[7] A. Shuchat, R. Shull, and A.N. Trenk. The fractional weak discrepancy of a partially ordered set. Discrete Applied Math., to appear.

[8] A. Shuchat, R. Shull, and A.N. Trenk. Range of the fractional weak discrepancy function. ORDER, 23:51-63, 2006.

[9] A. Shuchat, R. Shull, and A.N. Trenk. Fractional weak discrepancy and interval orders. In preparation.

[10] M. Skandera. A characterization of $(\mathbf{3}+\mathbf{1})$-free posets. J. of Combin. Theory, Ser. A., 93:231-241, 2001.

[11] R. Stanley. A symmetric function generalization of the chromatic polynomial of a graph. Adv. Math., 111:166-194, 1995.

[12] P.J. Tanenbaum, A.N. Trenk, and P.C. Fishburn. Linear discrepancy and weak discrepancy of partially ordered sets. ORDER, 18:201-225, 2001.

[13] A.N. Trenk. On $k$-weak orders: Recognition and a tolerance result. Discrete Math., 181:223-237, 1998. 